\title{
Ultrafast Electron Transfer in Photosynthesis: Reduced Pheophytin and Quinone Interaction Mediated by Conical Intersections
}

\author{
Gloria Olaso-González, Manuela Merchán, and Luis Serrano-Andrés* \\ Instituto de Ciencia Molecular \\ Universitat de València \\ Apartado 22085, ES-46071 Valencia, Spain \\ Gloria.Olaso@uv.es, Manuela.Merchan@uv.es, Luis.Serrano@uv.es,
}

\section{Supporting Information}

\footnotetext{
*To whom correspondence should be addressed. E-mail: Luis.Serrano@uv.es.
} 
Table S1. Cartesian coordinates of the ground-state geometry for FBC, optimized at the B3PW91/6-31G* level, within the constraints of $\mathrm{C}_{2 \mathrm{v}}$ point group (data in $\AA$ ).

$\begin{array}{lrrr}\mathrm{C} & 0.000000 & 2.890366 & 1.206130 \\ \mathrm{C} & 0.000000 & -2.890366 & 1.206130 \\ \mathrm{C} & 0.000000 & 1.099543 & -2.824949 \\ \mathrm{C} & 0.000000 & -1.099543 & -2.824949 \\ \mathrm{C} & 0.000000 & 1.081124 & 2.929490 \\ \mathrm{C} & 0.000000 & -1.081124 & 2.929490 \\ \mathrm{C} & 0.000000 & 0.766141 & -4.309521 \\ \mathrm{C} & 0.000000 & -0.766141 & -4.309521 \\ \mathrm{C} & 0.000000 & 4.246434 & 0.755421 \\ \mathrm{C} & 0.000000 & -4.246434 & 0.755421 \\ \mathrm{C} & 0.000000 & 4.236227 & -0.616078 \\ \mathrm{C} & 0.000000 & -4.236227 & -0.616078 \\ \mathrm{C} & 0.000000 & 0.679592 & 4.323464 \\ \mathrm{C} & 0.000000 & -0.679592 & 4.323464 \\ \mathrm{C} & 0.000000 & 2.419285 & 2.510553 \\ \mathrm{C} & 0.000000 & -2.419285 & 2.510553 \\ \mathrm{C} & 0.000000 & 2.411276 & -2.372596 \\ \mathrm{C} & 0.000000 & -2.411276 & -2.372596 \\ \mathrm{C} & 0.000000 & 2.871378 & -1.049796 \\ \mathrm{C} & 0.000000 & -2.871378 & -1.049796 \\ \mathrm{~N} & 0.000000 & 2.104663 & 0.076312 \\ \mathrm{~N} & 0.000000 & -2.104663 & 0.076312 \\ \mathrm{~N} & 0.000000 & 0.000000 & -2.043877 \\ \mathrm{~N} & 0.000000 & 0.000000 & 2.103204 \\ \mathrm{H} & 0.000000 & 1.090988 & 0.101046 \\ \mathrm{H} & 0.000000 & -1.090988 & 0.101046 \\ \mathrm{H} & 0.880339 & 1.190344 & -4.806153 \\ \mathrm{H} & -0.880339 & -1.190344 & -4.806153 \\ \mathrm{H} & -0.880339 & 1.190344 & -4.806153 \\ \mathrm{H} & 0.880339 & -1.190344 & -4.806153 \\ \mathrm{H} & 0.000000 & 5.108449 & 1.410210 \\ \mathrm{H} & 0.000000 & -5.108449 & 1.410210 \\ \mathrm{H} & 0.000000 & 5.087776 & -1.284202 \\ \mathrm{H} & 0.000000 & -5.087776 & -1.284202 \\ \mathrm{H} & 0.000000 & 3.177576 & 3.289192 \\ \mathrm{H} & 0.000000 & -3.177576 & 3.289192 \\ \mathrm{H} & 0.000000 & 3.189424 & -3.131395 \\ \mathrm{H} & 0.000000 & -3.189424 & -3.131395 \\ \mathrm{H} & 0.000000 & 1.352616 & 5.172927 \\ \mathrm{H} & 0.000000 & -1.352616 & 5.172927\end{array}$


Table S2. Cartesian coordinates of the ground-state geometry for FBC, optimized at the B3PW91/6-31G* level, within the constraints of $C_{\text {s }}$ point group (data in $\AA$ ).

$\begin{array}{rrrr}\mathrm{C} & -0.934236 & 2.989398 & 0.000000 \\ \mathrm{C} & 2.914309 & 0.834129 & 0.000000 \\ \mathrm{C} & 2.711353 & -1.355532 & 0.000000 \\ \mathrm{C} & -2.817244 & 1.346879 & 0.000000 \\ \mathrm{C} & -3.016804 & -0.806155 & 0.000000 \\ \mathrm{C} & 0.780307 & -2.955981 & 0.000000 \\ \mathrm{C} & -1.467764 & -2.766783 & 0.000000 \\ \mathrm{C} & -1.144069 & -4.158652 & 0.000000 \\ \mathrm{C} & 0.222517 & -4.275010 & 0.000000 \\ \mathrm{C} & 4.361869 & 0.365073 & 0.000000 \\ \mathrm{C} & 4.220458 & -1.160529 & 0.000000 \\ \mathrm{C} & -0.360256 & 4.298073 & 0.000000 \\ \mathrm{C} & 1.004440 & 4.161288 & 0.000000 \\ \mathrm{C} & -4.367593 & -0.277687 & 0.000000 \\ \mathrm{C} & -4.242155 & 1.075678 & 0.000000 \\ \mathrm{C} & -2.276564 & 2.640733 & 0.000000 \\ \mathrm{C} & 2.584999 & 2.182003 & 0.000000 \\ \mathrm{C} & -2.723138 & -2.177343 & 0.000000 \\ \mathrm{C} & 1.310315 & 2.762222 & 0.000000 \\ \mathrm{C} & 2.139917 & -2.619915 & 0.000000 \\ \mathrm{~N} & 0.118244 & 2.102755 & 0.000000 \\ \mathrm{~N} & 2.035074 & -0.188612 & 0.000000 \\ \mathrm{~N} & -0.270260 & -2.088667 & 0.000000 \\ \mathrm{~N} & -2.094242 & 0.194100 & 0.000000 \\ \mathrm{H} & 0.000000 & 1.095693 & 0.000000 \\ \mathrm{H} & -0.201428 & -1.077026 & 0.000000 \\ \mathrm{H} & -1.875583 & -4.956585 & 0.000000 \\ \mathrm{H} & 0.809241 & -5.184557 & 0.000000 \\ \mathrm{H} & 4.895421 & 0.741672 & 0.880545 \\ \mathrm{H} & 4.895421 & 0.741672 & -0.880545 \\ \mathrm{H} & -0.932661 & 5.216855 & 0.000000 \\ \mathrm{H} & 1.748333 & 4.947516 & 0.000000 \\ \mathrm{H} & -5.275623 & -0.869293 & 0.000000 \\ \mathrm{H} & -2.981878 & 3.467669 & 0.000000 \\ \mathrm{H} & 3.412403 & 2.886771 & 0.000000 \\ \mathrm{H} & -3.568428 & -2.860553 & 0.000000 \\ \mathrm{H} & 4.675698 & -1.628773 & -0.880545 \\ \mathrm{H} & 4.675698 & -1.628773 & 0.880545 \\ \mathrm{H} & -5.025978 & 1.824092 & 0.000000 \\ \mathrm{H} & 2.823689 & -3.464746 & 0.000000\end{array}$


Table S3. Cartesian coordinates of the ground-state geometry for $Q$, optimized at the $\pi$-CASSCF level, using the ANO-S basis set with the contraction scheme $\mathrm{C}, \mathrm{O}[3 \mathrm{~s} 2 \mathrm{p} 1 \mathrm{~d}] / \mathrm{H}[2 \mathrm{~s}]$ and within the constraints of $\mathrm{D}_{2 \mathrm{~h}}$ point group (data in $\AA$ ).

$\begin{array}{llrr}\mathrm{C} & 0.000000 & 0.000000 & 1.444813 \\ \mathrm{C} & 0.000000 & 0.000000 & -1.444813 \\ \mathrm{C} & 0.000000 & 1.270034 & 0.673993 \\ \mathrm{C} & 0.000000 & -1.270034 & 0.673993 \\ \mathrm{C} & 0.000000 & 1.270034 & -0.673993 \\ \mathrm{C} & 0.000000 & -1.270034 & -0.673993 \\ \mathrm{O} & 0.000000 & 0.000000 & 2.653992 \\ \mathrm{O} & 0.000000 & 0.000000 & -2.653992 \\ \mathrm{H} & 0.000000 & 2.184291 & 1.251504 \\ \mathrm{H} & 0.000000 & -2.184291 & 1.251504 \\ \mathrm{H} & 0.000000 & 2.184291 & -1.251504 \\ \mathrm{H} & 0.000000 & -2.184291 & -1.251504\end{array}$



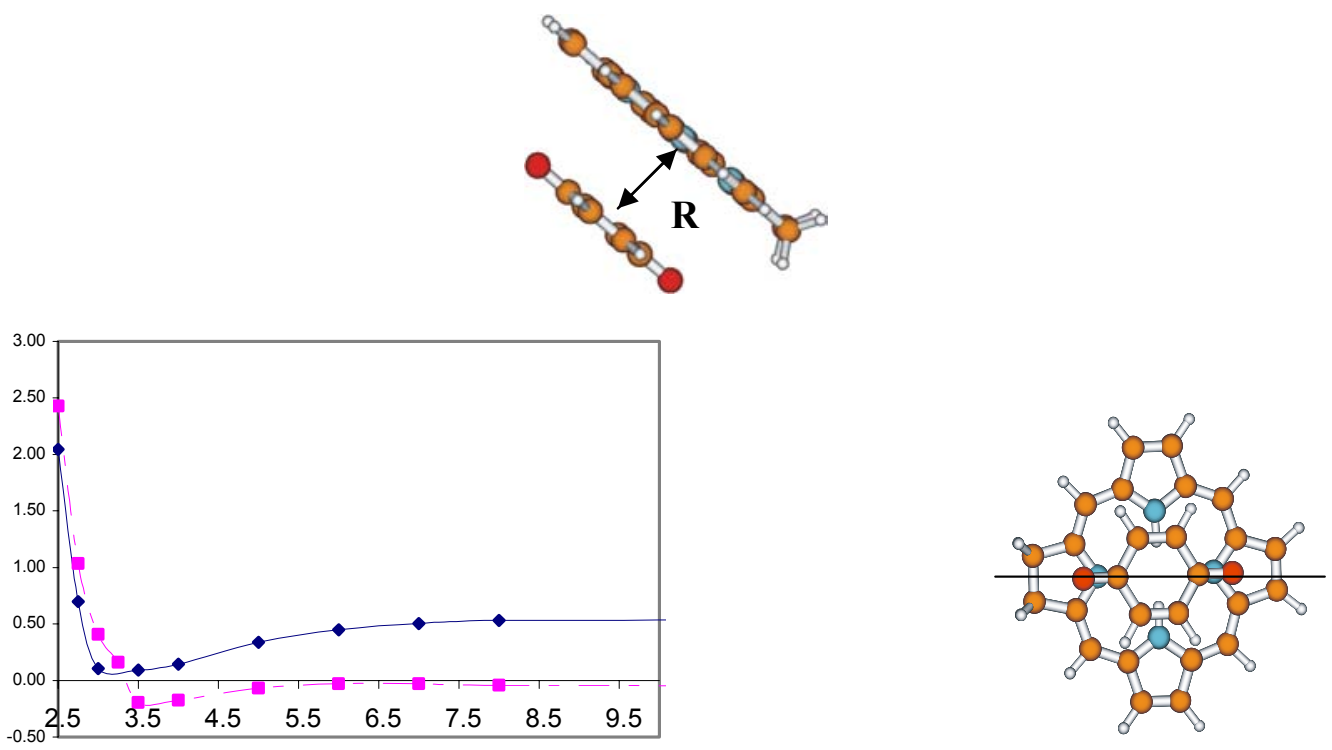

$\theta=0^{\circ}$
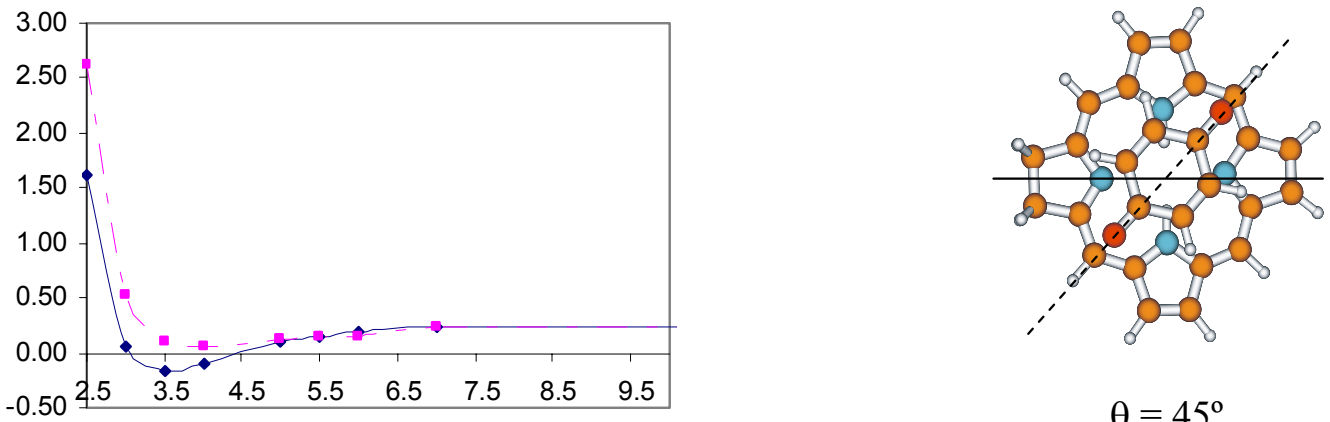

$$
\theta=45^{\circ}
$$
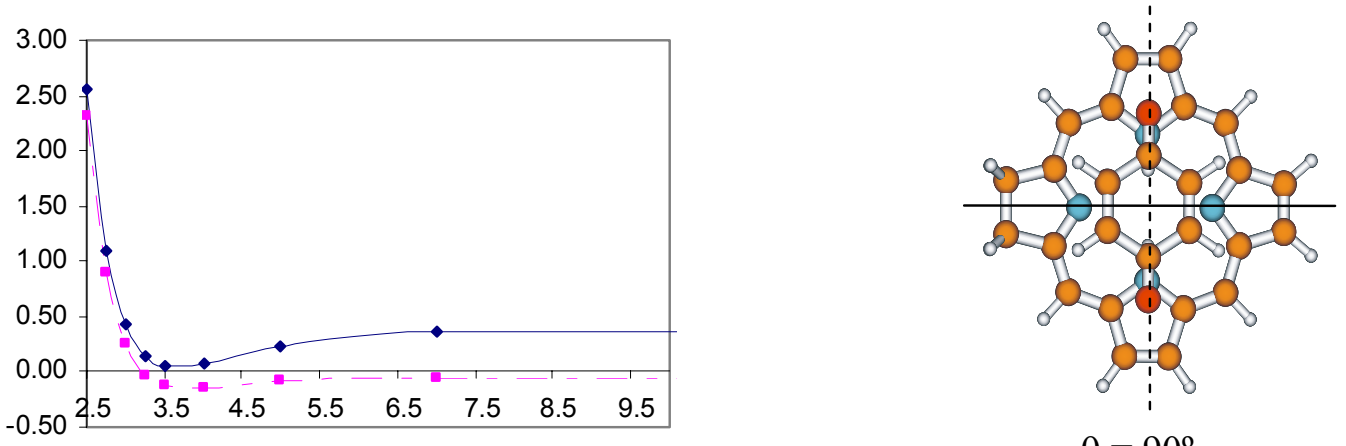

Figure S1. Potential energy curves (PECs) for $[\mathrm{FBC} / \mathrm{Q}]^{-}$along the intermolecular distance $R$ for the orientations defined by angle $\Theta$. The results have been obtained at the CASSCF/ANO-S C,N,O [3s2p1d]/H[2s] level. Energies referred to the subsystems at infinity separation. 